\title{
Konzeptionelle Kontinuität und biographischer Bruch
}

\author{
Ernst Schneider (1878-1957): Reform- \\ pädagoge, entlassener Lehrerbildner, \\ Universitätsdozent, Psychoanalytiker
}

Hans-Ulrich Grunder, Universität Basel

Im 19. Jahrhundert glich die Stelle des Direktors einer Lehrerbildungsstätte in der Schweiz einem Schleudersitz: Je nach politischer Konstellation der Regierung wurde sie besetzt oder ihr Inhaber wurde seines Amts enthoben. Im Kanton Bern ist dieser Vorgang durchgespielt worden, was illustriert, dass sich der Posten eines Seminardirektors an der Schnittstelle zwischen politischer und pädagogischer Macht befand. Hatte der Direktor des Seminars, für die pädagogische Richtung der Institution weitgehend allein verantwortlich, in der Regel einen Lehrauftrag an der Universität oder eine ausserordentliche Professur inne, oblag ihm dort auch die Aufgabe der universitären Ausbildung angehender Sekundarlehrer. Im Seminar der Primarlehrerausbildung prägte er, unterstützt von einem Konviktleiter, einem Methodiklehrer und Fachlehrern, alle Bereiche: von der strategischen Ausrichtung zu den Schulausflügen über die Prüfungen bis zum Stundenplan der angehenden Lehrkräfte und zum Unterricht. Bei Lehrplanrevisionen und Reglementsänderungen verfasste er die ersten Exposés. Bei wichtigen bildungspolitischen Entscheiden im Kanton konsultierte ihn die Regierung. Im übrigen stand er in engem Kontakt mit dem jeweiligen politisch Verantwortlichen, dem Unterrichts- oder Erziehungsdirektor, dessen Amtszeit in der Regel kürzer als die seine war-allerdings eben nicht immer, wie ein Blick in die Geschichte des Berner Staatsseminars und auf die Person Ernst Schneiders zeigt.

Im jenem Gebäude, wo Jahrzehnte später Sekundarlehramt und Pädagogisches Institut der Universität Bern beheimatet sein werden, wurde 1915 der damalige Seminardirektor von seinem Posten suspendiert, den er zehn Jahre zuvor erhalten hatte. Zu fragen ist: Was war geschehen? Welche Umstände und welche Anschuldigungen führten zu Schneiders Entlassung?

Ich schildere Schneiders beruflichen Lebensweg im Kontext reformpädagogischer Erneuerungsideen ${ }^{1}$, quellengestützt, in personen- und institutionengeschichtlicher Optik ${ }^{2}$, als Biographie 3 eines praktisch und wissenschaftlich tätigen Pädagogen und Lehrerbildners. Der «Reformpädagogik-Vorwurf» sollte (vergleichbar späterer Weggänge reformpädagogischer Protagonisten ${ }^{4}$ aus Lehrer- 
bildungsinstitutionen in der Schweiz, etwa von Oskar Messmer, Paul Häberlin oder Fritz Wartenweiler ${ }^{5}$ ) das Hauptmotiv für seine Entlassung sein, wogegen ich die damals geäusserte Behauptung - dies die These meiner Argumentation -, Schneider habe die angehenden Lehrkräfte am Seminar mit einem psychoanalytisch grundierten Unterricht ${ }^{6}$ in seinen Bann gezogen und unangemessen beeinflussen wollen, als wohlfeilen, aber verschleiernden Vorwand seiner damaligen Gegner einstufe. Vergleichbar mit der Position Wilhelm Reins in Deutschland, François Guex' an der Lausanner «Ecole normale» (Grunder, 1986a, 1993a) und jener Paul Conrads, des ehemaligen Lehrers an der Übungsschule Tuiskon Zillers in Leipzig und späteren Leiters des Churer Lehrerseminars, ist Schneider zu Beginn seiner beruflichen Karriere noch (moderater) Herbartianer (Schneider, 1898). Bald einmal aber (Schneider, 1905) wird er zum erklärten Exponenten einer «neuen Erziehung». Schneider sollte sich allerdings nicht, wie manche Protagonisten der damaligen «Schulerneuerungsbestrebungen» (Hinweise bei Herget, 1914; Bass, 1919; Karstädt, 1920; bezogen auf die Schweiz bereits sehr früh: Herzog, 1892; Balsiger, 1909, zusammenfassend), gegen die Professionalisierung der Lehrerschaft im Sinn Herbarts und für ein umfassendes Recht des Kindes auf Selbststeuerung und uneingeschränkte Selbsttätigkeit und auch nicht für daraus abgeleiteten radikalen Folgerungen, was die Lehrerbildung betrifft (Schneider, 1907), einsetzen.

\section{Das Lehrstück nach 1900: Professionalisierungsprozesse} in der Lehrerbildung

1908, drei Jahre nach Ernst Schneiders Amtsantritt als Direktor des deutschsprachigen Lehrerseminars des Kantons Bern, berichtet die zuständige Seminarkommission erstmals von den, wie sie schreibt, mangelhaften Kenntnissen der Zöglinge im Prüfungsfach Geschichte der Pädagogik, die anlässlich der Patentexamen konstatiert worden waren ${ }^{7}$. Ist der Seminarlehrplan nicht eingehalten worden?, fragen sich die Experten irritiert. Die Rüge an die Adresse des dreissigjährigen Ernst Schneider, der damals drei Jahre im Amt steht, lautet: «Wenn wir nun auch der Meinung sind, dass der Unterricht am Oberseminar Schritt halten soll mit den Neuerungen auf wissenschaftlichen Gebieten, so glauben wir doch verlangen zu dürfen, dass gleichwohl den bestehenden Vorschriften und Unterrichtsplänen nachgelebt werden soll...» ${ }^{8}$ Mit diesem ernüchternden Befund ist der «Berner Seminarhandel» lanciert. Politisch-pädagogisches Lehrstück, steht er für die Kontroverse zwischen «neuer» Schulreform und «alter» Schule.

\section{Schneiders berufliche Biographie}

Geboren am 17. Oktober 1878 in Bubendorf (BL), besucht Schneider die Primarschule, dann die Bezirksschule in Liestal. Er wird zum Primarlehrer am privaten Berner Lehrerseminar Muristalden ausgebildet und 1899 patentiert. In Innerberg/Wohlen (BE) wirkt er während zwei Jahren als Lehrer einer einklassigen Dorfschule, bezieht dann die Universität Bern, um Pädagogik, Philosophie, 
Geographie und Geschichte zu studieren, versieht Stellvertretungen an mehreren Stadt- und Landschulen und reist 1902 nach Jena, um sich bei Wilhelm Rein in Pädagogik speziell auszubilden. Nachdem er bei Rein eine "Pädagogische Fachprüfung» bestanden hat, arbeitet er als Lehrer an der Übungsschule des Pädagogischen Universitätsseminars, bevor ihm dort eine der Oberlehrerstellen angetragen wird ${ }^{9}$. Er akzeptiert, entschliesst sich aber bald (1903), in die Schweiz zurückzukehren und absolviert an der Universität Bern nach kurzem Studium bei Prof. Haag das Doktorexamen ${ }^{10} .1905$ wird Ernst Schneider Direktor des Bernischen Staatsseminars. Diese Stelle bekleidet er bis 1915. Nach seiner Suspension in Bern ist er, eingeladen von E. Claparède, als Dozent am Institut Jean-Jacques Rousseau in Genf tätig (1916-1920). 1920 nimmt Schneider einen Ruf auf den Lehrstuhl für Pädagogik an die Universität Riga an, den Pierre Bovet vermittelt hatte. Nach langen Auseinandersetzungen mit seiner Fakultät und der Universitätsleitung, so die wenigen Quellen aus der Universität, verlässt er Riga und Lettland, «because the faculty could not accept his extreme position in the field». ${ }^{11}$ Der Grund: Da die Unterrichtssprache an der Universität ab Mitte der Zwanzigerjahre nicht mehr deutsch, sondern lettisch war und man nur mit Zustimmung des Rektorats und einer ministeriellen Erlaubnis in einer anderen Sprache lehren durfte, demissioniert Schneider 1928. Die Genehmigung, in deutscher Sprache zu lehren, war 1928 nicht mehr erteilt worden - möglicherweise aufgrund politisch begründeter Animositäten mit Deutschland oder auf Druck lettischer Nationalisten.

Zwischen 1928 und 1946 betreibt Schneider in Stuttgart eine psychotherapeutische Praxis. Über diese Zeitspanne seiner Biographie ist bisher zu wenig bekannt (Schneider, 1956a, 1956b; Weber, 1999). Elf Jahre nach seiner Rückkehr in die Schweiz (1946) stirbt er in Muttenz an den Folgen eines Verkehrsunfalls (1957): Er wird auf dem Gehsteig von einem Auto angefahren und tödlich verletzt.

Welche Fakten führen die Demission Schneiders herbei? Welches sind die Folgen der Affäre? Wie hat sich das Verhältnis von pädagogisch-konzeptioneller Kontinuität und biographischem Bruch in Schneiders beruflicher Tätigkeit ausgeprägt?

Ein Blick in die Geschichte der bernischen Lehrerbildung (Grunder, 1993a) und der in ihr initiierten Reformen illustriert, dass sich am Lehrerseminar bereits vor der Ära reformerischer Aktivität, also seit 1860, Veränderungen (Unterricht, Schulleben, Theorie-Praxis-Bezug) ergeben haben - dass also die Kontroverse um Ernst Schneider eine lange Vorgeschichte aufweist.

Von den vorsichtigen Reformen Rüeggs und Martigs..., 1860 wird Hans-Rudolf Rüegg zum Direktor des Berner Staatsseminars in Münchenbuchsee gewählt (Kummer, 1874). Die erste Dekade seines Wirkens kennzeichnen Reformen, die den Unterricht prägen: Man richtet Musikzellen 
ein, hebt einen Badeweiher ${ }^{12}$ aus, schafft Apparate und Lehrmittel an und vergrössert die Bibliothek. Kurz nach seinem Amtsantritt lockert der Direktor die Disziplinvorschriften für die etwa hundertzwanzig angehenden Lehrer (ebd., 161). Knapp zwei Jahrzehnte später, am 16. Dezember 1879, bietet der Direktor in einem dreiseitigen Schreiben an, vom Amt zurückzutreten, da er als Professor für Pädagogik an die Berner Hochschule berufen worden ist. Seine Stelle wird am 22. Januar 1880 ausgeschrieben. Die «Zeit der sanften Seminarreformen» ist vorüber. Während der folgenden Periode verlangsamt sich das Reformtempo am Seminar Münchenbuchsee-Hofwil: Die «Zeit der zähflüssigen Reformen» setzt ein.

Wenngleich das Seminar in Münchenbuchsee während Rüeggs Zeit heftigen Angriffen ${ }^{13}$ und wachsender Konkurrenz ausgesetzt ist ${ }^{14}$, verfolgt Rüeggs Nachfolger, Pfarrer Emanuel Martig, zunächst den eingeschlagenen, mehrere Schlüsselkonzepte balancierenden Kurs ${ }^{15}$ : Lehrerbildung, so Martig, zielt auf "Gründlichkeit und denkende Verarbeitung des Gelernten» (Abschiedsfeier, 1880, S. 168) ab, sie soll nebst auf den Wissenserwerb ebenso auf ein sicheres, praktisch verwertbares Können, «zur Anwendung mit der Tat» (ebd., 168), hinwirken. Dies bedingt Martig zufolge, dass der angehende Lehrer den Bildungsstoff «selbsttätig» (ebd., 168) durchdringe und denkend verarbeite (ebd., 171)16.

In seinem der Unterrichtsdirektion eingereichten Stoffplan verlangt Martig ${ }^{17}$, der Stoff sei zu vermindern, so dass ihn die Seminaristen gründlicher verarbeiten können. Eine Erziehung zur vertieften geistigen Reife sei anzustrebendes Ziel. Erst im Winterhalbjahr 1891/1892, die Anstalt ist sieben Jahre zuvor nach Hofwil übergesiedelt, sollte sich, was den Stundenplan anbelangt, etwas verändern: Von nun an werden Handfertigkeit und Methodik doppelstündig erteilt. Zweimal nachmittags ist für die oberen Klassen nebst einem naturwissenschaftlichen Praktikum ein berufspraktischer Block von vier Stunden angesetzt. ${ }^{18}$

Im November 1881 setzt die kantonalbernische Unterrichtsdirektion den "Unterrichtsplan für das Deutsche Lehrerseminar», so wie ihn Martig vorgezeichnet hatte, in Kraft. Eingehend beschäftigt dort der Zielaspekt der Seminarausbildung: Der Unterricht im Seminar wolle, so liest man, den Zöglingen eine grundsätzliche, intellektuelle und sittlich-religiöse Ausbildung im Erwerb der erforderlichen Kenntnisse und Fertigkeiten gewähren, die für die Förderung der Volksbildung nötig seien (Unterrichtsplan, 1881).

Am 7. März 1900 ermächtigt die Regierung die Direktion, ein seit langem geplantes Vorhaben endlich zu verwirklichen: Sie verlängert die Seminarzeit der Berner Seminaristen von dreieinhalb auf vier Jahre. Während des ersten Drittels des Jahrs 1904 befindet sich das Seminar noch in Hofwil, zieht dann aber in die Stadt Bern um, provisorisch untergebracht im alten Hochschulgebäude. Auf die fünf neu zu besetzenden Lehrerstellen melden sich wohl aufgrund des nun attraktiver gewordenen Schulstandorts Dutzende von Bewerbern. Auch ein neuer Direktor wird gesucht. 
...über die umstrittene Wahl Schneiders zum Direktor des

Berner Staatsseminars...,

Am 3. Oktober 1905 wird das Oberseminar, in der Berner Länggasse im kurz zuvor fertiggestellten Gebäude, feierlich eröffnet. Martig hatte einige Monate vorher ${ }^{19}$ demissioniert, um einer jungen Kraft, wie er sagt, die Verantwortung zu übergeben. Martigs Nachfolger ist Ernst Schneider. Seine Wahl läutet die «Zeit der überstürzten Reformen» ein.

Trotz gewichtiger Einwände der skeptischen Wahlbehörde, der Seminarkommission, wird Schneider vom Regierungsrat gewählt. Dieser «Affront» veranlasst alle Kommissionsmitglieder, zu demissionieren ${ }^{20}$. Schneider ist, wenngleich sehr jung, bereits vor seinem Stellenantritt publizistisch aktiv gewesen: Fünf Jahre zuvor, als Zweiundzwanzigjähriger, hatte er selbstbewusst über die "Grundsätze der Herbart-Zillerschen Pädagogik» geschrieben (Schneider, 1898). Zum Seminardirektor gewählt, wird er zukünftig öffentlich und prononciert ihm wichtig scheinende Fragen der Pädagogik, v.a. der Lehrerbildung, thematisieren - dies zum Missvergnügen seiner zahlreichen Gegner im Kanton Bern. Anlässlich der Amtsübernahme hatte Schneider, in der Aula des neuen Schulgebäudes nach seinem Vorgänger Martig sprechend, sein pädagogisches Credo einer die Provokation vermutlich kaum begreifenden Schar von geladenen Gästen bekanntgegeben. Was dort zum Ausdruck kommt, ist frühe reformpädagogische Diktion: Der junge Direktor beschwört in pathetischen Worten die nach «Neugestaltung ringende, pädagogisch fruchtbare Zeit» und das "frohe, jugendfrische Schaffen, das vorwärts strebende Geistesleben», das ins Seminargebäude einziehen soll: «Nicht wiederkäuen! Geistige Durchbildung!» (Reden, 1905, S. 16). Zum einen muss der Lehrer philosophisch zu fragen lernen. Zum anderen hat Schneider zufolge die zeitgenössische kunstpädagogische Strömung gelehrt, dass Gemütsleben, Heiterkeit, Fröhlichkeit, Leben und Liebe zur Schule und auch zum Unterricht gehören. Das bedeutet für ihn freilich nicht, Schule sei grundsätzlich neu zu gestalten. Immerhin - so Schneider vorsichtig - kommen einige ihm zufolge neue Aspekte hinzu: Denn jedes Wissen sei erst dann echtes Wissen, wenn sich damit Gefühlsmomente verbänden (ebd., 19). Hierin liege der Schlüssel, womit das Überbürdungsproblem (Grunder 1993b, 2019) endlich zu beheben sei. Nicht in der quantitativen Reduktion, sondern im qualitativen Vertiefen liege der Zweck eines jeden Unterrichts, meint Schneider. Als Ziel seiner Pädagogik nennt er: «Bildung des Geistes, Bildung des Gemüts, Bildung des Willens zu einer geistig hochstehenden, weitblickenden Persönlichkeit, zu einer Lehrerpersönlichkeit, zu einem Künstler»(Reden, 1905, S. 21). Martig hatte Jahrzehnte zuvor mit beinahe denselben Worten Gleiches und mit entsprechenden Begriffen Vergleichbares ausgedrückt. Zu Beginn des neuen Jahrhunderts aber zeigen diese Formulierungen in Richtung einer «neuen Erziehung» und scheinen einen radikalen Umschwung zu implizieren. 
...zum Ausbruch des "Seminarstreits»...,

Schneider verändert aufgrund dieser Zielformel das Ausbildungssetting bis in den Seminaralltag: Nun wird ein Lesezimmer mit Zeitungen, Zeitschriften und Fachbüchern eingerichtet ${ }^{21}$. Dann weist der Stundenplan fortan einen freien Samstagnachmittag aus. Nachmittags ${ }^{22}$ werden nur noch Orgel-, Turn-, Zeichen- und Violinstunden - die musischen Fächer -, neben dem Besuch der Übungsschule, unterrichtet. Dagegen findet man die anderen Fächer - die intellektuell ausgerichteten - allesamt vormittags plaziert. Ein naturwissenschaftliches Praktikum für die oberen Klassen ist weiterhin verzeichnet. Als ein Ergebnis von Schneiders Studienreisen, die er während der folgenden Jahre unternimmt, darf der 1908 vorangetriebene Versuch der Seminardirektion gelten, für die Berner Lehrerbildungsstätte ebenfalls ein Schülerlaboratorium, wie es in den Seminaren von Wettingen und Küsnacht bereits besteht, zu fordern - seines praktischen, erzieherischen und pädagogischen Werts wegen, wie im Antrag ausgeführt wird. Laborarbeiten bildeten Schneider zufolge das notwendige Gegengewicht zu dem als mehr rezeptiv aufgefassten Unterricht in der Schulbank ${ }^{23}$.

Nach dem Abschluss des «Schneiderhandels» folgt am Berner Staatsseminar ab 1915 mit Direktor Johann Zürcher die "Zeit der administrativen Reformen». Der neue Direktor ${ }^{24}$ wird die Leitung des Seminars vierunddreissig Jahre lang (1916-1950) innehaben. Geschickt nimmt er die zuvor umstrittenen Fächer Pädagogik, spezielle Methodik und Psychologie aus der Debatte, indem er sie, was die Lehrerbildung anbetrifft, weitgehend delegitimiert und dergestalt der öffentlichen Diskussion entzieht. Die schulerneuernden Ansätze, wie sie am Berner Seminar Rüegg, Martig und Schneider realisiert hatten, sind in den Quellen bereits knapp sechs Jahre nach Schneiders Rücktritt nicht mehr sichtbar. Nach einer Reformphase am bernischen Staatsseminar, die ausgedehnte, kompliziert geschichtete und unübersichtliche Reformprozesse prägen, verlaufen die lange nur schleppend und dann rasant verwirklichten Reformen nun im Sand. War pädagogischer Wandel vor Zürchers Amtsantritt ein Diskussionsgegenstand, wurde er es nach dessen Wahl nie mehr, da sich der Direktor erfolgreich gegen jede Reformaktivität zur Wehr setzte.

\section{...den gehässigen Attacken...,}

Die Kampagne gegen Schneider, das sei im nachhinein vermutet, scheint von langer Hand orchestriert worden zu sein. Als «Hauptanklagepunkte» gelten die Diskussion der Psychoanalyse im Psychologieunterricht des Direktors und diejenige der experimentellen Pädagogik in seinem Pädagogikunterricht. Schneider wird Höhepunkte des Streits datieren auf die Jahre 1911 und 1915 - beschuldigt werden, er überfordere Jugendliche mit «ungereinigter Wissenschaft», welche die Seminaristen nicht zu begreifen imstande seien. Der unerfreuliche Prozess scheint seit 1908, mit dem ersten Brief der Seminarkommission also, zunächst versteckt zu verlaufen. Schneider ist dagegen machtlos, da in der Seminarkommission einige seiner Antagonisten sitzen, da die Presse die Neuigkeiten aus dem 
noch jungen Staatsseminar genüsslich aufbereitet und weil die Lehrerverbände nicht vor verleumderischen und unzutreffenden Aussagen zurückschrecken. Über "Anklageschriften», "Beschwerden» (vgl. Beschwerde, 1911), "Umfragen bei Lehrern», «Inspektionen bei Schneider-Schülern», «Expertisen», Unterschriftensammlungen, Lehrerzusammenkünften, Schneiders Gegenwehr (Schneider, 1911) sowie Pressequerelen sind bis heute erst spärliche, nicht wirklich erhellende Informationen publiziert worden (Schärrer, 1979; Weber, 1999).

Der Vorwurf, mit den Seminaristen Psychoanalyse betrieben zu haben und sie «verdorben» zu haben, greift, nach der Durchsicht der Unterlagen, zu kurz. Ebenso ungenügend untermauert ist der Angriff seitens der Schneider-Gegner, die belegen möchten, die abgehenden Seminaristen seien keine tüchtigen Lehrer. Dagegen sprechen die Inspektionsberichte ${ }^{25}$. Zweifelsohne ist jedoch gegen Schneider nun endlich ein Vorwand gefunden, der es erlaubt, einen, den «modernen Strömungen der Pädagogik» positiv gegenüberstehenden Direktor zu stürzen: Der unbelegte Vorwand lautet: Schneiders Behandlung psychoanalytischer Sachverhalte im Unterricht. Das tragfähigere Motiv erkenne ich in Schneiders Engagement gegen einen herbartianistisch akzentuierten, also für einen eher kindorientiert arrangierten, auf selbständigem Lernen beruhenden Unterricht, der in einer lernfreundlichen Atmosphäre verlaufen und der die Balance zwischen sinnvoll ausgewählten Inhalten, optimierten Lernprozessen und einem zweckdienlichen, also den Lernstoffen angepassten Medieneinsatz nicht ignorieren wollte.

1911 gibt der Regierungsrat laut den gedruckten Quellen vermittelnd zu, Schneider habe sich zwar einer Amtspflichtverletzung nicht schuldig gemacht. Sein Eifer zugunsten einer «sogenannten Schulreform» sei «an und für sich» als berechtigt zu anerkennen. Der Direktor lasse im Unterricht indessen öfters «die nötige Klarheit vermissen» und habe sich "da und dort in der Wahl seiner Mittel vergriffen» 26 . Deswegen wird Schneider 1911 lediglich provisorisch wiedergewählt. Seine definitive Wiederwahl für weitere sechs Jahre erfolgt am 10. September 1912, als die Angelegenheit ihre Brisanz verloren zu haben scheint.

Noch 1908 hatte die Seminarkommission Schneider gestützt, was ihr Engagement für die Wahl eines von Schneider portierten Methodiklehrers - in mehreren Schriftstücken «R.» ${ }^{27}$ genannt - zeigte. Was der von der Kommission verwendete Passus «Richtung von R.» meint, sind «R.s» reformerisch orientierte Methoden in der Lehrerbildung. Hatte die Kommission diese eben noch verteidigt, folgen ihre davor warnenden Worte kurz darauf. Weil die Gegner der «neuen Linie» schliesslich zusammenfinden, wird «R.» vorderhand nicht definitiv gewählt. Anhand eines Berichts, den Schneider für 1910 versprechen muss, und der über den «Kurs am Seminar» Auskunft geben soll, will man entscheiden, ob die «Vertretung der neuen Tendenzen am Seminar noch zu verstärken sei» 28 . Die Wahl des neuen Methodiklehrers verringert die pädagogischen Vorbehalte gegen Schneider zumindest seitens der Übungslehrer, wie Schneider ausführt: «Heute habe ich die Genugtuung, dass in den Hauptpunkten Zustimmung herrscht.»29 
Mit seinem Text «Klare Kampfstellungen», gegen einen bekannten Schulinspektor gerichtet (Kasser, 1911), hatte Schneider das Feuer jedoch nochmals entfacht (Schneider, 1911), vermutlich ohne sich der zu erwartenden Konsequenzen wirklich gewahr zu sein. Die Reaktionen auf diese Schrift erfolgen unmittelbar.

Schneider wird vorgeworfen, seine Anwürfe gegen die Lehrerschaft schürten den Zorn der Betroffenen. Er habe am Bildungswesen und der Schule «vernichtendste Kritik» (Beschwerde, 1911, 2) geübt, folge "deutschen Schriftstellern» (ebd., 2) minderwertigen Rangs und habe mit der Gründung der «Berner Seminarblätter»30, einem reformorientierten pädagogischen Periodikum, Keile in die Lehrerschaft getrieben. Schneider hätte «die Jungen dadurch ferngehalten, vom Einfluss der Alten» (Beschwerde, 1911, 2) zu profitieren. Die Folgen sind für die Schneider-Kritiker unbestreitbar: «Von da an trohnte am Staatsseminar das künstlerisch-ästhetische Bildungsideal» (ebd., 3), womit eine seitens der Gegner nicht näher spezifizierte «neue Erziehung» gemeint sein muss. Schneiders Handeln stelle einen Akt verständnisloser Undankbarkeit dar, zumal er unterstelle, nur «künstlerisch-ästhetische Suggestion» (ebd., 5) fördere das Gute und nachhaltig beteuere, moralisches Einwirken auf das Kind sei veraltete Praxis. Eines wird zumindest zugestanden: «Dass er das Arbeitsprinzip in der Schule in den Vordergrund stellt, wollen wir gelten lassen. Es ist ja schon seit Jahrzehnten eine allgemeine pädagogische Forderung, gar nichts Neues. Es kann sich ja nur darum handeln, es im Unterrichte allgemeiner zur Geltung zu bringen. Dafür braucht man aber keine «klaren Kampfstellungen»« (ebd., 6). Zwar sei die Idee der Arbeitsschule nützlich und brauchbar, dagegen das «künstlerischästhetische Ideal», wonach "das humane, das religiös-menschliche Erziehungsprinzip ersetzen soll» (ebd., 7) eben nicht: «Mit dem Schlagwort der freieren Entwicklung der Persönlichkeitswerte im Kinde hat Herr Dr. Schneider in den Köpfen der jungen Lehrer schon ungemein viel Verwirrung angestiftet.» (Ebd., 7) Schneiders als «schöne Theorie» lächerlich gemachter Gedanke, knapp zusammengefasst und formuliert durch die Brille seiner Gegner: «Wer dem Kinde nicht freien Spielraum lässt, wer ihm seine Geistesprodukte korrigiert, damit hineinpfuscht, erzieht es zur geistigen Lüge. Nur was das Kind aus sich heraus, aus innerem Antriebe schafft, hat bildenden Wert. Weg mit Zwang, Disziplin, ewiger Nörgelei und Korrektur! So lautet die schöne Theorie des neuen Bildungsideals.» (Ebd., 7) Die zitierten «Belege» stammen aus den «Berner Seminarblättern» und werden im Wortlaut abgedruckt. Die Textstellen aber verweisen allenfalls darauf, dass Schneider für eine Schulreform plädiert, also zugunsten einer angemessenen Selbsttätigkeit des Kindes hinsichtlich seiner Lernprozesse. Die umfangreiche Pressedokumentation ${ }^{31}$ veranschaulicht, worum es geht: Um die öffentlich von Schneider vertretene, aber keineswegs umstürzlerische schulerneuernde Position des Direktors. Diese hinterrücks inszenierte Hetze gegen den Pädagogen sei kindisch und feig, schreibt die Berner Volkszeitung am 7. März $1911^{32}$.

...Schneiders entschiedener Gegenwehr..., 
Die Replik der Verteidiger Schneiders fokussiert auf dessen Prämissen: Das «neue Bildungsideal» trage zum selbständigen Fühlen, Werden und Wollen des Zöglings bei und erfordere neue Unterrichtsmethoden. Sachlich gehen Schneiders Helfer mit ihren Gegnern ins Gericht, indem sie deren Thesen sorgfältig und ausführlich bearbeiten. Bei einigen Aussagen gelingt es ihnen zu belegen, dass die Nachweise zugunsten der gegnerischen Optik aus Schneiders Texten willkürlich herausgepickt und neu zusammengefügt worden sind. Andere Behauptungen vermögen sie zu widerlegen - als frech unterstellt, unsachlich oder übertrieben. Daraus schliessen sie, dass es zwar um Schneider gehe, aber in erster Linie doch um die kontinuierliche Reform der Lehrerbildung und der Schulen im Kanton, was die Schneider-Gegner scharf zurückweisen. Indem sie Schneider angriffen, so die Befürworter, sollen Schulreformen gar nicht erst keimen können, geschweige denn, was schon von ihnen da sei, sich weiterzuentwickeln wagen. Den Voten entnimmt man, dass Schneiders Supporter befürchten, das Seminar Bern werde in «pädagogischer Provinzialität» versinken, setze sein attackierter Direktor seine Arbeit nicht fort. An ihm bemängle man lediglich, dass er gewisse Ideen 33 in die Schweiz gebracht oder hier aktualisiert habe und nachhaltig vertrete. Von Psychoanalyse, einem in anderen Texten bemühten Aspekt (s.o.), ist bemerkenswerter Weise hier nicht mehr die Rede, was die Sicht untermauert, Schneider sei nicht vornehmlich deswegen angegriffen worden. Allerdings mag der Umstand, dass er 1910 bei Oskar Pfister und Carl Gustav Jung eine Lehranalyse absolviert hatte und die Publikation eines Beitrags von Oskar Messmer mit dem Titel «Die Psychoanalyse und deren pädagogische Bedeutung" in den "Berner Seminarblättern» dazu geführt haben, dass er verdächtigt wurde, einen psychoanalytisch gefärbten Unterricht erteilt zu haben (Messmer, 1911/1912; Moll, 1997, S. 136).

\section{...dem halbherzigen Support, dem offenen Lob, erneutem Zwist...}

Einer der moderateren Gegner, Dr. K. Fischer, Lektor für Methodik am Seminar, «beweist» in seinem Aufsatz, Schneider habe insgesamt gar keinen neuen «und namentlich keinen greifbaren Vorschlag für irgendwelche Art der Schulreform» vorgelegt. Was er empfehle, sei nachgerade bekannt: sowohl der «freie Aufsatz» 34 , der Appell, das Schulbuch als Volksbuch zu verwenden als auch der entwicklungspsychologische Ansatz in der Kinderpsychologie.

Allmählich kühlt die Kontroverse wieder aus.

Die Teilnahme an der Landesausstellung in Bern (1914) illustriert dann aber, dass sich Schneider nachdrücklich schulreformerisch, reformpädagogisch, engagiert: Seine Schule ist dort «durch eine möglichst abgerundete Vorführung der Bestrebungen des Seminars zur Erziehung durch Selbstbetätigung» (Regierungsrat, 1915, 124) vertreten, heisst es im Bericht der Direktion des Unterrichtswesens. Der unverdächtige Berichterstatter der «Schweizerischen Lehrerzeitung», Seminardirektor Conrad aus Chur, vermerkt enthusiastisch und den Gang der Reformen am Seminar illustrierend, es werde «da vorgeführt, wie die 
Seminaristen zu Hofwil und Bern arbeiten und lernen» (ebd., 124). Conrad fährt, Schneider lobend, fort:

Wir sehen die Hofwiler Zöglinge im Garten und auf der Wiese beschäftigt, wir sehen sie Kartoffeln graben und Holz sägen und dann wieder sich im Weiher beim Bad tummeln. Dann erhält eine Klasse Deutsch, eine andere Geschichte im Park zu Hofwil, eine Berner Klasse Stillehre im Berner Münster, eine weitere Klasse skizziert im Freien ein vor ihr stehendes Pferd. Die Seminaristen beim Wandtafelzeichen, beim Modellieren, bei praktischen Übungen im Samariterkurs, beim Bau physikalischer Apparate, im physikalischen und botanischen Praktikum, bei einem Besuch der Gasfabrik, auf einer Reise im Gebirge, schwerbeladen über einen Gletscher schreitend, dann wieder voll Leben und Fröhlichkeit bei den Zelten: Das sind weitere Bilder von bestrickendem Reiz, die uns so recht eindringlich lehren, wie vielseitig man die jungen Leute in diesen Schulen betätigt, und wie sehr man sich abmüht, überall aus den besten Quellen zu schöpfen, direkt aus Natur und Leben und nicht bloss aus toten Büchern.[...] Schulen, die die neuen pädagogischen Anschauungen [...] mit dieser Vielseitigkeit durchführen, gibt es jedenfalls wenige. Wir sind deshalb dem Berner Seminardirektor zu grossem Dank verpflichtet, dass er sich die Mühe gegeben hat, uns einen wichtigen Teil des Lebens in seinen Anstalten in so trefflicher Weise vor Augen zu führen. (Ebd., 124)

Bereits ein Jahr später flackern die Scharmützel um Schneider erneut auf: Die Direktion für das Unterrichtswesen will nun prüfen, «ob wir mit der Art und Weise der Ausbildung unserer jungen Primarlehrer auf dem richtigen Wege sind» 35 . "Im wohlgemeinten Bestreben, alle neueren und neuesten Strömungen der pädagogischen Wissenschaft für seinen Unterricht zu verwenden» (ebd., 32), sei Schneider «auf Abwege gekommen» (ebd., 32), hatte die Direktion für das Unterrichtswesen in ihrem Verwaltungsbericht zuvor schon angemerkt.

Endgültig eingeleitet wird die zweite Etappe des «Seminar-Handels», wie Zeitgenossen die unerfreuliche Angelegenheit auch nennen, als der Unterrichtsdirektor eine Expertenkommission bestellt, die zu evaluieren hat, «ob die Leitung und der Unterricht am Staatsseminar den berechtigten Anforderungen an eine zweckmässige Lehrerbildung gerecht werden» 36 .

\section{...und der endgültigen Suspension}

Die Mitglieder der Kommission ${ }^{37}$ erörtern die Fakten, die Anwürfe gegenüber Schneider und sein Bemühen, ihnen standzuhalten, anlässlich einer vierstündigen Zusammenkunft. Sie erarbeiten einen Bericht, der am 23. Dezember 1915 beim Treffen der Seminarkommission von Unterrichtsdirektor Lohner kommentiert wird ${ }^{38}$. Bemerkenswert ist an diesem Text der Hinweis, weshalb «ein längeres Verbleiben von Dr. Schneider und Dr. Röthlisberger am Seminar unmöglich sei» ${ }^{39}$. Dort fällt ebenfalls auf, dass nun unvermittelt «die Psycho- 
analyse» gegen Schneider erneut ins Feld geführt wird. Nicht oder nur als Nebensache angedeutet, wird dagegen die «neue pädagogische Grundorientierung», die Schneider ins Seminar gebracht haben soll:

Aus dem Bericht gewinnt man die Überzeugung, dass Dr. Schneider sich in der sog. Psychoanalyse auf Irrwegen befindet. Das sexuelle Leben wird als Ausgangspunkt genommen und alles läuft auf das Geschlechtsleben hinaus. Dieses Fach ${ }^{40}$ der Psychoanalyse könnte allenfalls an der Hochschule von Akademikern doziert werden, und zwar von Lehrern, die das ganze Gebiet vollständig beherrschen würden. Dr. Schneider aber, der dieses Gebiet jedenfalls nicht beherrscht, übermittelt es unverarbeitet den jungen Seminaristen, die noch weniger davon verstehen. Daraus können grosse Verheerungen entstehen. Erfahrungen nach dieser Seite sind bereits gemacht worden. Regierungsrat Lohner verliest zwei Briefe von jungen Lehrern, [...] deren brieflicher Verkehr mit ihren Schulkommissionen usw., als Frucht der Schneiderschen Schule bezeichnet werden können. ${ }^{41}$

Was ihm beträchtliche Sorgen bereite «seien die Folgen, die aus diesen psychoanalytischen Versuchen entstehen könnten», merkt der Präsident der Seminarkommission an: «Dr. Schneider hat darin gefühlt, dass er noch strittige, unfertige Theorien als fait accompli seinen Schülern servierte.» Gegen Schneider sprechen sich auch die Herren Rickli und Schär, beide Mitglieder der Expertenkommission, aus: Schneider sei seiner Aufgabe nicht gewachsen, sexuell pathologisch veranlagt, Argumenten unzugänglich, von sich überzeugt und sofort abzuberufen, empfehlen sie übereinstimmend. Für den verantwortlichen Politiker Lohner ist es - und dieser Satz wird letztlich im Protokoll aber durchgestrichen, bleibt allerdings noch lesbar - «ein Unglückstag für den Kanton Bern gewesen, als Dr. Schneider zum Seminardirektor gewählt wurde» 42 .

Aufgrund des Berichts der Expertenkommission 43 ist Regierungsrat Lohner überzeugt, «dass ein längeres Verbleiben von Dr. Schneider und Dr. Röthlisberger am Seminar unmöglich sei» ${ }^{44}$. Die Vorwürfe gegenüber Schneider sind nun wieder dieselben wie 1911: Er favorisiere die Psychoanalyse ${ }^{45}$ und die Lehrer bereite er lediglich lückenhaft auf ihren späteren Beruf vor. 1915 wird er nicht mehr wiedergewählt.

\section{Fazit: Der "Schneiderhandel" als Beispiel für eine Kontroverse zwischen althergebrachter Pädagogik und Schulreformambitionen}

Schneider, der engagierte und nachdrücklich schulreformerisch-reformpädagogisch denkende und handelnde Seminarleiter, bringt die von Rüegg und Martig initiierten Reformen am Berner Staatsseminar voran. Er wird allerdings kurz nach seiner Wahl schon zur Zielscheibe einer schulpolitischen, pädagogischen und unterrichtsmethodisch munitionierten Verfolgungsjagd. Speziell aussagekräftig in diesem langdauernden, zähfliessenden «Handel» sind die zahlreichen 
Antworten der damals befragten Ehemaligen, der betroffenen «Schneiderschüler». Sie waren die Schulinspektoren gleichsam zu verhören gezwungen ${ }^{46}$ : Die selbstgefällig-offenherzigen und auch verräterischen Inspektorenberichte erschliessen die Schwierigkeiten junger Lehrer, wenn sie den beruflichen Alltag zu bewältigen haben, weniger dagegen etwaige Fehler der Ausbildungsinstitution. Zuweilen monieren die Inspektoren geringfügige Details, Nachlässigkeiten von Novizen im Beruf, die auf mangelnde Einsicht, Unerfahrenheit oder unzureichende Ordnungsliebe zurückzuführen sind. Der Fächer ist weitgespannt: Den Absolventen des Seminars wird insbesondere vorgeworfen, sie achteten wenig auf die Körperhaltung ihrer Schülerinnen und Schüler, sie wollten die "Kinder zum Denken und zu freier, selbsttätiger Arbeit» anregen (!), sie liessen es an «rechter Geisteszucht ermangeln», sie machten «Waldschule» und Spaziergänge, unterrichteten im Freien, legten Stunden zusammen, ein Lehrer unterrichte während eines ganzen Sommers täglich zwei Stunden Botanik, hielten zu wenig von schulmeisterlichem Drill, begingen methodische Fehler, wollten die Lehrmittel nicht verwenden, nähmen die Vorschriften nicht ernst und betätigten die Kinder «künstlerisch». Ein Inspizient gibt indessen zu, es sei trotz aller Kritik ein frischer Geist in die Schule eingezogen, wenngleich die Lehrer zuviel Selbstvertrauen und den Kopf «voller wirrer Reformideen» hätten.

Charakteristisch für die Art, wie der verhandelte Gegenstand angegangen wird, sind die Rapporte der Patentprüfungskommission und der Examinatoren für das Fach Pädagogik. Den zu patentierenden Seminaristen wird zwar bescheinigt, ihre Kenntnisse, was den pädagogischen und psychologischen Bereich betreffe, seien auf dem neuesten Stand. Die Historische Pädagogik und die Schulgesetzgebung des Kantons Bern, beides Lerngegenstände, beachteten sie aber zu wenig. ${ }^{47}$ Die Grundsätze würden zwar gelehrt, aber zuhanden der Praxis werde eben zu wenig geleistet, wenngleich Schneider bemüht sei, die Jünglinge zum Denken anzuregen. Man führe zwar in befriedigender Manier in die Problematik des Fachs ein, wenngleich zuweilen über die Köpfe der Schüler hinweg geredet werde. Trotz allem werde versucht, für die Schule Enthusiasmus zu wecken, konzediert die Patentprüfungskommission.

Schneider bekämpft die Attacken der beiden Kommissionen unverzüglich: Die Disziplin sei besser geworden, gerade weil die Schüler mehr Vertrauen zuerkannt erhielten - ein Zusammenhang, den auch Martig exponiert hatte. Die Gegner bemühten darüberhinaus Einzel-fälle und zitierten oberflächlich. Schneider hält ausdrücklich an der Neuorganisation der Patentexamen fest, die er als Intelligenz-, nicht als Wissensprüfung interpretiert, und besteht auf mit in die Examensnote einfliessenden «Erfahrungsnoten». Der Direktor fügt den Streit schliesslich in einen weitgefassten Rahmen ein: Der gegenwärtig tobende Schulkampf, so schreibt er, sei ein «Ausschnitt aus den allgemeinen Schulkämpfen der Gegenwart. Diese sind bekannt geworden unter dem Namen: Schulreform.» ${ }^{48}$ Leider debattiere man hierzulande die schulreformerischen Programme kaum. Er hingegen vertrete eine sachlich-befürwortende und prüfende Position, ohne 
dass seine Person für die Reform stünde. Bedauerlicherweise hätten seine Gegner von der «ganzen grossen pädagogischen Bewegung der Gegenwart nicht viel» 49 kennengelernt, fügt er ironisch an. Kern der Opposition seien die Exponenten des pädagogisch eher konservativen «Berner Schulblattes», das ungewohnte didaktische Methoden sich zu diskutieren weigere. 50

In seinen Verteidigungsschriften, die zwar inhaltlich Position beziehen, in der Regel aber in einem vorsichtigen, defensiven Duktus verfasst sind (z.B. Schneider, 1911) hat der Direktor sowohl vor wie nach dem «Berner Seminarhandel» pädagogische Themen so offen abgehandelt, dass der schulreformerische Akzent seines Unterrichts daraus ableitbar wird (Schneider, 1907, 1908, 1907-1915, 1956a). 51

In seinem Beitrag «Vom Aufsatz» (Schneider, 1908), worin er seinen reformaffinen Standpunkt herausstreicht, unterscheidet Schneider den grammatikalischen und stilistischen vom freien Aufsatz, den (wenngleich unter anderen pädagogisch-programmatischen Prämissen) nach 1918 auch Célestin Freinet in Bar-sur-Loup propagierte. Schneider opponiert gegen den «vorbereiteten Aufsatz» und empfiehlt eine Aufsatzreform. Diese muss vornehmlich die «rote Gefahr» kritisch beachten - jenen Lehrer, der die Schülerarbeit mit dem roten Stift pedantisch und demotivierend verändere und korrigiere.

Erst 1956, mit dem Erscheinen der Autobiographie Schneiders (1956a), tritt ein bislang hier nicht gewürdigter Aspekt zum Streit um den kritisierten Direktor hinzu: Die Angehörigen der freisinnigen Partei hätten gegen seinen Stellenantritt protestiert, weil er am Evangelischen Privatseminar Muristalden bei Bern zum Lehrer ausgebildet worden sei, wird angemerkt. Seine Reformen am Staatsseminar, gerade die Reisen, Doppelstunden, Praktika, andersartig gewichteten Examen, seine Opposition gegen den «Paradeaufsatz» sowie der Aufbau der «Berner Seminarblätter» hätten die Schulinspektoren negativ reagieren lassen (Schneider 1956a, S. 63). Andere Direktoren von Lehrerseminaren in der Schweiz haben im übrigen dasselbe Schicksal erfahren (s.o., Grunder, 1993a): Fritz Wartenweiler, Leiter der solothurnischen Lehrerbildung, Opfer des «Seminarstreits» von 1917, wo es um die Notengebung anlässlich der Patentprüfung ging, und Paul Häberlin, Direktor des Kreuzlinger Lehrerseminars, abberufen 190852. Erwähnenswert ist ebenfalls die Affäre um die Entlassung Oskar Messmers in Rorschach (1915).

Obgleich über dreihundertfünfzig Lehrer im Kanton Bern Schneider schriftlich unterstützen, wird er 1915 abgesetzt, nachdem Röthlisberger schon im Januar 1915 seine Demission eingereicht hatte. Die Schneider-Gegner dürften aber erst im nachhinein eingesehen haben, dass Schneiders Ansatz jener gewesen ist, der eine moderne Lehrerbildung hätte konstituieren können. Dass zwischen Rüegg, Martig und Schneider hingegen eine stetige Entwicklung feststellbar ist, was die Reformen am Berner Seminar anbelangt, ist bislang weitgehend unbeachtet geblieben. In dieser Optik ist Schneider trotz allem Reformenthusiasmus 
lediglich der entschlossene Ausgestalter einer ab 1860 allmählich einsetzenden Reform der Ausbildung am deutschsprachigen Lehrerseminar im Kanton Bern, die aber erst nach der Jahrhundertwende entscheidend akzeleriert verlaufen ist, bevor sie nach 1915 abrupt gestoppt wurde.

\section{Anmerkungen}

1 Zum Forschungsstand zur europäischen Reformpädagogik, insbesondere zu den zahlreichen Variationen erziehungs-, bildungs- und schulreformerischen Denkens und Handelns: vgl. Barz, 2018; Mayer, 2018; Skiera, 2018; zur Definition, zu Charakteristika und zu Implikationen des Begriffs «Reformpädagogik»: vgl. Grunder, 2015; Oelkers, 2005; Ullrich \& Idel, 2017.

2 Zum forschungsmethodologischen Ansatz: Es geht hier um eine biographisch-realgeschichtliche Betrachtung. Allerdings verzichte ich an dieser Stelle darauf, die sozialhistorischen Hintergründe und die mentalitätsgeschichtlichen Aspekte der Situation in der deutschsprachigen Schweiz zu Beginn des 20. Jahrhunderts auszuleuchten, währenddem ich institutionengeschichtliche Aspekte ebenfalls anspreche; zum Verhältnis von Reformpädagogik und pädagogischer Reform: vgl. Grunder, 2015; Hansmann, 2005.

3 Schneider hat eine Autobiographie verfasst: Schneider, 1956a.

4 Zur Reformpädagogik in der deutschsprachigen Schweiz: Criblez, 1995; Gronert \& Schraut, 2018; Grunder, 2007, 2015.

5 Zum Verhältnis von damaliger Lehrerausbildung und Reformpädagogik: Grunder, $1993 \mathrm{a}$.

6 Hinweise zur damaligen Debatte um den Einfluss der Psychoanalyse auf die Erneuerung der Erziehung: Bühler, 2013, 2014, 2018.

7 Vgl. Schneiders Beitrag zur Positionierung der historischen Pädagogik, eines seiner bevorzugten Themen (Schneider, 1956a), am Lehrerseminar: Schneider, 1907.

8 Brief vom 30. April an die Direktion des Unterrichtswesens, SBE, Akten folgende.

9 Eine Oberlehrerstelle hatte zwischen 1892 und 1895 Hermann Lietz innegehabt (Grunder, 1987).

10 Schneiders schulhistorische Dissertation ist bis heute lesenswert: Er wertet darin unter einer architekturhistorischen und modernitätstheoretischen Perspektive die Frage 15 (a bis d) zum Schulhausbau der Stapfer-Enquête von 1800 aus: Schneider, 1905.

11 So die Aussagen von Prof. Dr. Iveta Kestere und Prof. Dr. A. Zigmunde (2015/2016) nach dem Studium der Archive gegenüber H.U. Grunder; vgl. auch Weber, 1999.

12 Rüegg möchte Schwimmunterricht erteilen lassen.

13 Insbesondere gegen den Religionsunterricht.

14 Gründung des Evangelischen Seminars auf dem Muristalden und der Neuen Mädchenschule in Bern, beide unter "pietistischer Leitung» (Abschiedsfeier zu Ehren, 1880, S. 163).

15 Ausgewogener Theorie-Praxis-Bezug, wissenschaftlich-theoretische Ausbildung, berufspraktische Anteile, spezielle Methodik (Fachdidaktik) je Unterrichtsfach, Pädagogik und Psychologie als «Leitfächer», vertiefte «Schulkunde».

16 Entsprechend, aber in weitergefasstem Zusammenhang: Müllener, 1892.

17 Am 14. August 1880, SEB, Akten 1871-1881.

18 Mit Brief vom 15. April 1881 (SEB, Akten 1871-1881) hatte Martig dem Unterrichtsdirektor mitgeteilt, dass er die Fächer Pädagogik und Religion übernehmen würde.

19 Brief Martigs an die Direktion des Unterrichtswesens vom 18. Januar 1905, SBE, Akten folgende.

20 Am 18.4.1905.

21 Brief Schneiders an die Direktion des Unterrichtswesens vom 31. Oktober 1905, SBE, Akten folgende.

22 Im Sommer 1906. 
23 Brief Schneiders an die Direktion des Unterrichtswesens vom 30. Juli 1908, SBE, Akten folgende.

24 Zürcher (1878-1962), gleich alt wie Schneider, Primar-, Sekundar- und Gymnasiallehrer, hatte sich 1904 auch auf die Stelle des Seminardirektors beworben. Zürcher hat nicht publiziert; eine selbständige Veröffentlichung Zürchers fehlt.

25 SBE, Seminarhandel.

26 Brief des Regierungsrats an die Seminarkommission vom 13. September 1911, SBE, Akten folgende; vgl. Grunder, 2015.

27 Gemeint ist Dr. Röthlisberger.

28 Brief der Seminarkommission an die Direktion des Unterrichtswesens vom 23. April 1909, SBE, Akten folgende.

29 Brief Schneiders an die Seminarkommission vom 1. März 1909, SBE, Akten folgende.

30 Schneider gründet die «Berner Seminarblätter» 1907 (Grunder, 1986b). Ab 1912 ediert er das Periodikum gemeinsam mit O. Messmer, O. Pfister und O.v.Greyerz (Schneider, 1907-1915).

31 SBE, Seminarhandel.

32 Ebd., 2.

33 "... die gegenwärtig im Schwange sind...»

34 Gutachten über die untersuchten Beiträge Schneiders in den «Berner Seminarblättern» vom 21. März 1911, 3.

35 Brief der Direktion des Unterrichtswesens an die Primarschulinspektoren vom 20. Mai 1915, SBE, Akten folgende.

36 Brief der Direktion des Unterrichtswesens vom 25. August 1915 an die Primarschulinspektoren, SBE, Akten folgende.

37 Zusammensetzung: s.u.

38 SBE, Seminarkommission, 1915.

39 Protokoll der Sitzung vom 23. Dezember 1915, vgl. auch SBE, Seminarkommission.

40 Am Seminar existiert kein «Fach» oder «Schulfach» Psychoanalyse. Psychoanalytische Sachverhalte müsste Schneider also in dem von ihm unterrichteten Fach «Pädagogik» erörtert haben. Dass er die Schüler in diesem Kontext psychoanalytische Inhalte bearbeiten liess oder sie gar "psychoanalytisch» beeinflusst haben könnte, erachte ich als unwahrscheinlich.. Allerdings sei - gegen die These dieses Beitrags - konzediert (Moll, 1997), dass Schneider seinen Pädagogikunterricht mit psychoanalytischen Einsprengseln «angereichert haben könnte» (ebd., S. 137, S. 138), worüber er aber erst vierzig Jahre später berichten wird; vgl. Schneider, 1956b.

41 Protokoll der Sitzung vom 23. Dezember 1915, S. 2, SEB, Seminarkommission.

42 Protokoll der Sitzung vom 23. Dezember 1915, S. 3-5, SEB, Seminarkommission.

43 In ihr sitzen zwei 1905 abgewiesene Mitbewerber Schneiders um das Direktorenamt, zwei Schulinspektoren, ein Beamter und ein Oberlehrer.

44 Protokoll der Sitzung der Seminarkommission vom 23.12.1915, SEB, Seminarkommission.

45 «Irrwege»; Protokoll der Sitzung der Seminarkommission vom 23.12.1915, S. 3, SEB, Seminarkommission.

46 Die Inspektorenberichte sollten das Abbild der pädagogischen Grundsätze präsentieren, wie sie am Seminar hochgehalten wurden. Von Psychoanalyse ist darin nicht die Rede...

47 Diesen Vorwurf sollte Schneider entkräften; er hatte sein Konzept der Vermittlung historischer Pädagogik bereits früh vorgelegt: vgl. Schneider 1907.

48 Brief Schneiders an die Direktion des Unterrichtswesens vom 11. Juni 1911, SBE, Akten folgende.

49 Brief Schneiders an die Direktion des Unterrichtswesens vom 11. Juni 1911, S. 3, SBE, Akten folgende. 
50 In Schneiders Optik sind Werkstätten, Labors, ein Exkursionskredit, eine eigene Übungsschule sowie eine Neudefinition der Patentabschlüsse unabdingbar.

51 Unter den ehemaligen Schülern Schneiders engagieren sich im Berner Seminarstreit für den Direktor unterschiedliche Charaktere: Ernst Nobs, der nachmalige Bundesrat, Hans Müller, der spätere Führer der Jungbauern, Paul Hulliger, der Initiant der Schriftreform, der Publizist Hans Mühlestein, Fritz Schwarz, Sekundarlehrer und Freiwirtschaftsbefürworter, Fritz Marbach, Mitarbeiter Fritz-Jean Begerts, sowie Otto von Greyerz, damals Lehrer am Landerziehungsheim Glarisegg, und Johannes Itten, der nachmalige Leiter des Vorkurses des Staatlichen Bauhauses in Weimar.

52 Der Rücktritt als Seminardirektor erfolgte 1909 als Folge einer Kontroverse, die aufgrund des Widerstands des Lehrerkollegiums gegenüber Häberlins Reformvorschlägen entbrannt war.

\section{Literaturverzeichnis}

\section{Archivalien}

Archivalien im Staatsarchiv des Kantons Bern. Bern, Aktengruppe BBIII.

Berichte des Regierungsrates über die Staatsverwaltung des Kantons Bern (Auszüge aus dem Verwaltungsbericht der Erziehungsdirektion. Bern 1870 ff. (zit. als: Regierungsrat, 1870 ff.).

\section{Literatur}

Abschiedsfeier. (1880). Abschiedsfeier zu Ehren der Herren Rüegg und Langhans. Bern: Schmidt. Balsiger, E. (1909). Schulreform-Bestrebungen der Gegenwart. Zürich: o.V.

Barz, H. (Hrsg.). (2018). Handbuch Bildungsreform und Reformpädagogik. Wiesbaden: Springer VS.

Bass (von Salten), A. (1919). Deutsche Schulreform. Beiträge zum deutschen Schul- und Erziehungswesen. Leipzig: Leipziger Verlags- und Kommisionsbuchhandlung.

Beschwerde (1911). Beschwerde an die tit. Unterrichtsdirektion des Kantons Bern über die Tätigkeit des Herrn Dr. E. Schneider (hrsg. von einem Initiativkomitee). Bern: H. Jent \& Co.

Bühler, P. (2013). Unterrichten mit Gefühl - Psychoanalytische Pädagogik zu Beginn des 20. Jahrhunderts. Jahrbuch für historische Bildungsforschung, 19, 247-261.

Bühler, P. (2014). Der «letzte Zufluchtsort für Stürmer und Dränger»? Psychoanalytische Pädagogik in den "Berner Seminarblättern" und der "Schulreform» 1907-1930. Schweizerische Zeitschrift für Bildungswissenschaften, 36(1), 51-65.

Bühler, P. (2018). Neue Formen des Heils und der Heilung. Zur Psychopathologie des Schullebens am Anfang des 20. Jahrhunderts. In Bühler, P. \& Bühler, T. (Hrsg.), Sakralität und Pädagogik (S. 173-193). Bern: Haupt.

Criblez, L. (1995). Reformpädagogik in der Krise: Krise der Reformpädagogik. Bildungsforschung \& Bildungspraxis 17(2), 194-209.

Gronert, M., \& Schraut, A. (Hrsg.). (2018). Handbuch Vereine der Reformpädagogik, überregional arbeitende reformpädagogische Vereinigungen sowie bildungsentwicklerisch initiative Einrichtungen mit Brückenfunktion in Deutschland, Österreich, der Schweiz, Südtirol und Liechtenstein. Baden-Baden: Ergon.

Grunder, H. U. (1986a). Von der Kritik zu den Konzepten. Aspekte einer Geschichte der Pädagogik der französischsprachigen Schweiz im 20. Jahrhundert. Frankfurt a. M.: Haag \& Herchen.

Grunder, H. U. (1986b). Die «Berner Seminarblätter». Schweizerische Lehrerzeitung, 133(6), 14-17.

Grunder, H. U. (1987). Das schweizerische Landerziehungsheim. Frankfurt a. M.: Peter Lang. Grunder, H. U. (1993a). Seminarreform und Reformpädagogik. Bern: Peter Lang.

Grunder, H. U. (1993b). Schule als Überbürdung - überbürdete Schule: Und die Schulpäd- 
agogik? SGBF-Bulletin (Publikationsorgan der Schweizerischen Gesellschaft für Bildungsforschung), 2, 4-15.

Grunder, H. U. (2007). Anarchistische Erziehung als libertäre Reformpädagogik. Baltmannsweiler: Schneider.

Grunder, H. U. (2015). Schulreform und Reformschulen. Bad Heilbrunn: Klinkhardt.

Grunder, H. U. (2019). Jenseits der Fächer? Anforderungen an die Schule, ihre Motive, ihre Ursachen und ihre Folgen - Lektionen aus der schweizerischen Schulgeschichte. In K. Zierer. (Hrsg.), Jahrbuch für Allgemeine Didaktik (S. 53-64). Baltmannsweiler: Schneider Hohengehren.

Hansmann, O. (2005). Reformpädagogik oder pädagogische Reform. Aachen: Shaker.

Herget, A. (1914). Die wichtigsten Strömungen im pädagogischen Leben der Gegenwart. Prag: A. Haase.

Herzog, J. A. (1892). Die Schule und ihr neuer Aufbau auf natürlicher Grundlage. Zürich: Schmidt.

Karstädt, O. (1920). Methodische Strömungen der Gegenwart. Langensalza: Beltz.

Kasser, E. (1911). Schulfragen. Bern: Francke.

Kummer, J. J. (1874). Geschichte des Schulwesens im Kanton Bern. Bern: Wyss.

Mayer, S. M. (2018). Die Reformpädagogik in der aktuellen Kontroverse: eine Metaperspektive. Unveröffentlichte Dissertation, Universität Augsburg.

Messmer, O. (1911/1912). Die Psychoanalyse und deren pädagogische Bedeutung. Berner Seminarblätter, 5, 283-292.

Moll, J. (1997). Psychoanalytische Diskussion im Wiener Kreis um Freud. Das Interesse der Pädagogik an der Psychoanalyse. In H. Badertscher \& H. U. Grunder (Hrsg.), Geschichte der Erziehung und Schule in der Schweiz im 19. und 20. Jahrhundert (S. 127-164). Bern: Haupt.

Müllener, C. (1892). Beiträge und Vorschläge zur Reorganisation der Lehrerbildung auf pädagogischer Grundlage. Bern: Schmid, Francke \& Co.

Oelkers, J. (2005). Reformpädagogik: eine kritische Dogmengeschichte (4. vollst. überarb. Aufl). München: Juventa.

Reden. (1905). Reden gehalten an der Eröffnungsfeier. Bern: Gustav Grunau.

Schärrer, M. (1979). Dr. E. Schneider als Seminardirektor in Bern. Unveröffentlichte Hausarbeit, Universität Zürich.

Skiera, E. (2018). Reformpädagogik in Geschichte und Gegenwart: eine kritische Einführung (reprint). Berlin: Oldenbourg.

Schneider, E. (1898). Über die Grundsätze der Herbart-Zillerschen Pädagogik. Berner Schulblatt, 32, 5-8.

Schneider, E. (1905). Die bernische Landschule am Ende des 19. Jahrhunderts. Bern: G. Grunau.

Schneider, E. (1907). Die historische Pädagogik am Seminar. Schweizerische Pädagogische Zeitschrift, 17, 275-284.

Schneider, E. (Hrsg.). (1907-1915). Berner Seminarblätter. Zeitschrift für Schulreform. Bern: Grunau, Suter.

Schneider, E. (1908). Vom Aufsatz. Berner Seminarblätter, 2(2), 22-25.

Schneider, E. (1911). Klare Kampfstellungen. Berner Seminarblätter, 4(10), 413-417.

Schneider, E. (1956a). Aus meinen Lern- und Lehrjahren. Bern: Pestalozzi-Fellenberg-Haus.

Schneider, E. (1956b). Wie ich zu Freud und zur Psychoanalyse kam. Schweizerische Zeitschrift für Psychologie und ibre Anwendungen, 15(2), 131-133.

Ullrich, H., \& Idel, T.-S. (Hrsg.). (2017). Handbuch Reformpädagogik. Weinheim: Beltz.

Unterrichtsplan (1881). Unterrichtsplan für das Deutsche Lehrerseminar des Kantons Bern. Biel: Schüler.

Weber, K. (1999). "Es geht ein mächtiges Sehnen durch unsere Zeit». Reformbestrebungen der Jahrhundertwende und Rezeption der Psychoanalyse am Beispiel der Biografie von Ernst Schneider 1878-1957. Bern: Peter Lang. 
Schlagworte: Reformpädagogik, Reformpädagogik in der Schweiz, Psychoanalyse, Schulreform, Lehrerbildung

\section{Continuité conceptuelle et rupture biographique Ernst Schneider (1878-1957): un partisan de l'Éducation nouvelle, formateur d'instituteurs congédié, professeur d'université, psychanalyste}

\section{Résumé}

Au $19^{\mathrm{e}}$ siècle, le poste de directeur d'École normale en Suisse ressemblait à un siège éjectable. Selon la constellation politique cantonale, il était embauché ou relevé de sa fonction. La position de directeurs d'École normale se trouvant au croisement du pouvoir politique et pédagogique, ce modèle a été appliqué plusieurs fois dans le canton de Berne. Le directeur d'une École normale était responsable de presque tout, en particulier des directives pédagogiques, du choix des méthodes, de l'engagement des maîtres formateurs comme du fonctionnement du quotidien. Il était aussi répondant de toute l'organisation: l'enseignement, les excursions, les examens, les horaires. Quand des plans d'études ou règlements devaient être modifiés, c'est encore lui qui s'en chargeait. Il était aussi consulté par le ministre cantonal pour toutes les questions concernant l'éducation ou l'école publique. Cette situation s'est prolongée au $20^{e}$ siècle. Le cas d'Ernst Schneider illustre la précarité de la position d'un directeur d'École normale en ce temps-là.

Mots-clés: Éducation nouvelle, Éducation nouvelle en Suisse, Psychanalyse, réforme de l'école, formation des instituteurs

\section{Continuità concettuale e spaccatura biografica: \\ Ernst Schneider (1878-1957): sostenitore della scuola attiva, formatore d'insegnanti d'istituto, professore universitario, psicanalista}

\section{Riassunto}

Nel 19e secolo il posto di direttore di una scuola normale in Svizzera era come un seggiolino eiettabile. Tale figura professionale era incaricata o destituita a seconda della costellazione politica del governo cantonale. Nel canton Berna questa dinamica si è verificata diverse volte, in quanto il posto di direttore di una scuola normale si situava al crocevia tra il potere politico e pedagogico. Il direttore di una scuola normale era responsabile di quasi tutto e, in particolare, delle direttive pedagogiche, della scelta dei metodi, dell'impiego dei maestri nella scuola normale e del suo funzionamento quotidiano. Egli era anche garante di tutta l'organizzazione dell'istituto: insegnamento, escursioni, esami, orario. 
Quando si dovevano modificare i piani di studio o i regolamenti, era lui che ne elaborava i contenuti. Egli era consultato dal ministro della cultura cantonale per tutte le questioni relative all'educazione e alla scuola. Questa situazione si è protratta nel 20e secolo. Il caso di Ernst Schneider dimostra la precarietà che caratterizza la posizione di direttore di una scuola normale nella parentesi cronologica studiata.

Parole chiave: Educazione progressiva, educazione progressiva in Svizzera, psicoanalisi, riforma della scuola, formazione degli insegnanti

\title{
Conceptual continuity and biographical discontinuity Ernst Schneider (1878-1957): reform pedagogue, dismissed teacher trainer, university lecturer, psychoanalyst
}

\begin{abstract}
In the 19th century, the position of director of a teacher training instititon in Switzerland was similar to an ejection seat: depending on the political constellation of the government, it was occupied or its holder was removed from office. In the Canton of Berne, this process has been played through, which illustrates that the post of a director of teacher seminar was at the interface between political and pedagogical power. The director of the seminar, who was largely solely responsible for the pedagogical direction of the institution, usually held also a teaching position at the university or an extraordinary professorship: He was therefore also responsible for the university training of future secondary teachers. In the primary teacher training seminar, supported by a lecturer, a methodology teacher and teachers in different school subjects, he shaped all areas: from strategic orientation to school excursions, from examinations to the timetable of future teachers and also teaching. He wrote the first exposés for curriculum revisions and changes in regulations. He was consulted by the government on important educational policy decisions in the canton. He was also in close contact with the respective politically responsible person, the minister of education, whose term of office was generally shorter than his - but not always, as a glance at the history of the Bern State Seminar and the person of Ernst Schneider shows.
\end{abstract}

Keywords: Progressive Education, Progressive Education in Switzerland, Psychoanalysis, School Reform, Teacher Training 
Grunder, Hans-Ulrich, Prof. em. Dr. phil. I habil., 1954, Sekundarlehrerstudium und Unterrichtstätigkeit; Gründung einer alternativen Schule (1978); Zweitstudium (Pädagogik, Ethnologie Journalismus); Assistent am Pädagogischen Institut der Universität Bern, Promotion, Habilitation; Vizedirektor der Sekundarlehrerausbildung an der Universität Bern;

Ordinarius für Schulpädagogik an der Universität Tübingen (D) (1995-2005); Professor für Pädagogik an der Pädagogischen Hochschule FHNW und der Universität Basel (2005-2014); Direktor des Instituts für Bildungswissenschaften der Universität Basel (ab 1.1.2015, bis 31.1.2019).

Thürliacker 41, CH-3033 Wohlen bei Bern

E-Mail: hansulrich.grunder@unibas.ch 